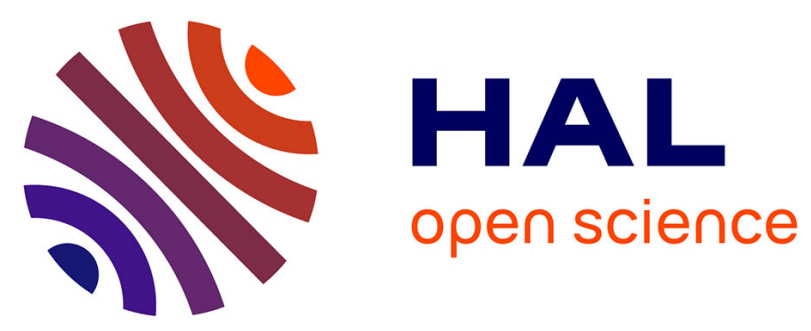

\title{
Inventaire, fréquence et agressivité des différentes espèces ou variétés de Fusarium responsables de la pourriture sèche des tubercules de pomme de terre
}

Bernard Tivoli, Bernard Jouan, Marie-Thérèse Sanson, Emile Lemarchand

\section{- To cite this version:}

Bernard Tivoli, Bernard Jouan, Marie-Thérèse Sanson, Emile Lemarchand. Inventaire, fréquence et agressivité des différentes espèces ou variétés de Fusarium responsables de la pourriture sèche des tubercules de pomme de terre. Agronomie, 1981, 1 (9), pp.787-794. hal-00884325

\section{HAL Id: hal-00884325 \\ https://hal.science/hal-00884325}

Submitted on 1 Jan 1981

HAL is a multi-disciplinary open access archive for the deposit and dissemination of scientific research documents, whether they are published or not. The documents may come from teaching and research institutions in France or abroad, or from public or private research centers.
L'archive ouverte pluridisciplinaire HAL, est destinée au dépôt et à la diffusion de documents scientifiques de niveau recherche, publiés ou non, émanant des établissements d'enseignement et de recherche français ou étrangers, des laboratoires publics ou privés. 


\title{
Inventaire, fréquence et agressivité des différentes espè- ces ou variétés de Fusarium responsables de la pourriture sèche des tubercules de pomme de terre
}

\author{
Bernard TIVOLI \& Bernard JOUAN \\ avec la collaboration technique de Marie-Thérèse SANSON et Emilc LI:MARCHAND \\ I.N.R.A., Station de Pathologie végétale, Centre de recherches de Rennes, F 35650 Le Rheu.
}

\section{RÉSUMÉ \\ Fusarium, \\ Pomme de terre, \\ Inventaire, \\ Fréquence, \\ Agressivité.}

Plusieurs espèces ou variétés de Fusarium sont isolées de tubercules de pomme de terre présentant des symptômes de pourriture. $F$. roseum var. sambucinum, $F$. solani var. coeruleum, $F$. roseum var. arthrosporioides sont les espèces ou les variétés les plus fréquemment représentées. Des souches de $F$. roseum var. graminearum, de $F$. roseum var. culmorum, de $F$. roseum type $\mathrm{X}$ et de $F$. oxysporum sont également isolées.

Si $F$. roseum var. sambucinum et $F$. solani var. coeruleum semblent constituer des agents de pourriture sèche très dangereux dans les conditions climatiques de notre pays (l'optimum du développement de la maladie se situe aux environs de $\left.15^{\circ} \mathrm{C}\right) ; F$. roseum var. arthrosporioides peut provoquer des pourritures à partir de $18-20^{\circ} \mathrm{C} . F$. roseum var, graminearum et $F$. roseum var. culmorum occasionnent, quant à eux, des dégâts à des températures voisines de $25-30^{\circ} \mathrm{C}$. Le rôle parasitaire de $F$. roseum type $\mathrm{X}$ et de $F$. oxysporum n'est pas clairement établi puisqu'ils provoquent peu (ou pas) de nécroses sur tubercules.

\section{SUMMARY \\ Fusarium, \\ Potato, \\ Importance, \\ Frequency, \\ Pathogenicity.}

\begin{abstract}
Importance and pathogenicity of the different Fusarium species and varieties causing dry-rot in potato tubers

Fusarium isolation from dry-rot potato tubers of 1843 samples harvested in 1978 and 1956 samples harvested in 1979 was carried out.

F. roseum var. sambucinum proved to be the predominating species in $35-40$ p. 100 of the samples; the species second in frequency were $F$. solani var. coeruleum and $F$. roseum var. arthrosporioides; lastly, $F$. roseum var. culmorum, $F$. roseum var. graminearum, $F$. roseum type $\mathrm{X}$ and $F$. oxysporum were also found. As to their pathogenicity, $F$. roseum var. sambucinum was found to be highly pathogenic (from 5 to $30^{\circ} \mathrm{C}$ ); $F$. solani var. coeruleum, after a slow establishement on the potato tubers, is very pathogenic at $15^{\circ} \mathrm{C}$. F. roseum var. arthrosporioides is pathogenic from $18{ }^{\circ} \mathrm{C}$ to $30^{\circ} \mathrm{C}$. These three Fusarium species or varieties can be considered as the most important causal agents of dry rot of potato tubers. $F$. roseum var. culmorum and $F$. roseum var. graminearum are pathogenic only at $25-30^{\circ} \mathrm{C}$. F. roseum type $\mathrm{X}$ and $F$. oxysporum are slightly pathogenic to apathogenic.
\end{abstract}

\section{INTRODUCTION}

Les parasites agents de pourriture des tubercules de pomme de terre (Fusarium sp., Phoma sp., Erwinia sp.) sont susceptibles de provoquer, certaines années, d'importantes pertes pendant la conservation du plant (BOYD, 1972). Le développement de ces agents est favorisé par les blessures occasionnées lors de la récolte ou des opérations de manutention en vue du stockage et de la commercialisation. Les derniers travaux relatifs à la fusariose de la pomme de terre en France sont dus à LANSADE (1950). Cette maladic est alors attribuée à Fusarium coeruleum (Lib.) Sacc. Plus récemment, Tivoli et al. (1979) ont mis en ćvidence, dans les locaux de conservation, un ensemble d'espèces et de variétés de Fusarium dont le rôle parasitaire n'a pas été précisć. A l'étranger, les nombreux travaux réalisés montrent que plusieurs espèces ou variétés de Fusarium sont susceptibles de provoquer une pourriture sèche des tubercules. Ainsi, Mc KEE (1954), en GrandeBretagne, attribue la maladie à $F$. coeruleum $(93$ p. 100 des cas), F. avenaceum (Fr.) Sacc. (6 p. 100 des cas) et $F$. arthrosporioides Sherb. ou $F$. tricinctum (Corda) Sacc. (1 p. 100 des cas) ; Boyd (1972) relate qu'en Amérique du Nord, la maladie est due surtout à $F$. sulphureum Schel et, pour une moindre part, à $F$. coeruleum et $F$. trichothecioides Wollenw. Enfin, plus récemment PETT \& GOTZ (1978) notent qu'en Allemagne de l'Est, des isolements réalisés à partir de tubercules présentant des symptômes en cours d'évolution donnent 79 p. 100 de $F$. sulphureum, 13 p. 100 de $F$. coeruleum, les autres Fusarium étant constitués de $F$. avenaceum et $F$. culmorum (W. G. Smith) Sacc. Il semble donc, d'après ces quelques exemples, que chaque pays ou 
chaque région du globe, héberge des populations pathogènes de Fusarium qui leur sont plus ou moins spécifiques.

Une réactualisation des connaissances sur les agents de pourriture fusarienne en France s'impose afin de pouvoir mieux orienter les travaux de recherche destinés à améliorer les possibilités de lutte. Ce travail se propose d'examiner les différents agents de fusariose rencontrés, d'étudier leur fréquence et de préciser leur agressivité.

\section{MATÉRIEL ET MÉTHODES}

\section{Fréquence des différentes espèces et variétés de Fusarium}

Des échantillons d'environ 10 tubercules présentant des symptômes de pourriture sèche lors de la conservation sont prélevés dans différentes localités; 30 p. 100 d'entre-eux appartiennent au cultivar «Bintje» (cultivar le plus répandu en France), le reste étant constitué de nombreux autres cultivars dont les principaux sont "Claustar", «Apollo », «Sirtema » et «Ostara ». Les isolements sont effectués sur milieu gélosé à base d'extrait de malt à 2 p. 100 à raison de 5 boîtes de Petri par échantillon. Ils ont permis d'obtenir 1843 isolats de Fusarium en 1978 et 1456 isolats de Fusarium en 1979.

\section{Détermination des espèces ou variétés de Fusarium}

La nomenclature adoptée dans cette étude est celle de MEssiaen \& CASSini (1968) et de Messiaen et al., (1976). Les différentes espèces citées ci-dessus deviennent dans cette nomenclature :

F. coeruleum: F. solani var. coeruleum (Sacc.) Booth

$F$. avenaceum: F. roseum var. avenaceum (Sacc.) Sn. et $\mathrm{H}$.

F. arthrosporioides: $F$. roseum var. arthrosporioides (Sherb.) Messiaen et Cassini.

F. tricinctum : F. tricinctum (Cda) Sn. et $\mathrm{H}$.

F. sulphureum: F. roseum var. sambucinum (Fuck.) Sn. et $\mathrm{H}$.

F. culmorum : F. roseum var. culmorum (Schwabe) Sn. et $\mathrm{H}$.

F. graminearum : F. roseum var. graminearum (Schwabe) Sn. et $\mathrm{H}$.

La détermination s'effectue après repiquage de chacune des colonies isolées sur 2 milieux gélosés: un milieu complet à base d'extrait de pomme de terre (P.D.A., $40 \mathrm{~g} / \mathrm{l}$ ) et un milieu minéral additionné de glucose (milieu de TANAKA-OU, 1972- modifié selon BOISSONNETMENES \& LECOQ, 1976). Après incubation des colonies $\left(23^{\circ} \mathrm{C}\right.$, éclairement de 10000 lux avec une photopériode de $12 \mathrm{~h}$ ), l'identification des Fusarium est réalisée en faisant appel aux divers critères macro- et microscopiques habituels. Les colonies présentant sur les 2 milieux des différences de morphologie, la détermination est facilitée. Ainsi, sur le milieu Tanaka, $F$. solani var. coeruleum développe sa pigmentation azurée tandis que $F$. roseum var. arthrosporioides se colore en rouge carmin avec un mycélium aérien blanc très dense. La différence d'aspect des colonies de cette dernière variété de Fusarium sur PDA et sur Tanaka permet sa distinction des autres variétés de Fusarium roseum. Enfin le milieu Tanaka induit chez $F$. roseum type $\mathrm{X}$, décrit par MESSIAEN et al. (1976), la formation de chlamydospores caractéristiques de ce type alors que cette induction est absente chez $F$. roseum var. culmorum. $F$. roseum type X, décrit par MESSIAEN et al. (1976), est caractérisé en outre par des macroconidies « plus pointues et plus busquées à l'extrémité, plus minces à la base ", que celles de $F$. roseum var. culmorum et par des chlamydospores très abondantes et «d'allure analogue à celle des equiseti ».

\section{Les tests d'inoculation sur tubercules}

Les tests d'agressivité des isolats sont réalisés sur des tubercules entiers du cultivar "Bintje" récoltés depuis environ 3 mois et conservés dans un local réfrigéré à $4{ }^{\circ} \mathrm{C}$. Ces tubercules désinfectés, par trempage dans l'alcool pendant $30 \mathrm{~s}$, sont séchés puis blessés dans leur partie médiane, à l'aide d'un emporte-pièce permettant de réaliser un puits d'un diamètre et d'une profondeur de $5 \mathrm{~mm}$.

L'inoculation consiste à déposer dans chaque puits une pastille colonisée par le champignon. Les tubercules ainsi inoculés sont mis en incubation à une température de $15^{\circ} \mathrm{C}$ (température optimale pour le développement des pourritures de Fusarium selon BoYD, 1972). Trois semaines plus tard, on note après avoir sectionné les tubercules selon leur grand axe passant par le point d'inoculation, les caractéristiques de la nécrose : largeur et profondeur. Les mesures présentées dans les résultats sont mentionnées après déduction de $5 \mathrm{~mm}$ correspondant au puits initial (fig. 1).

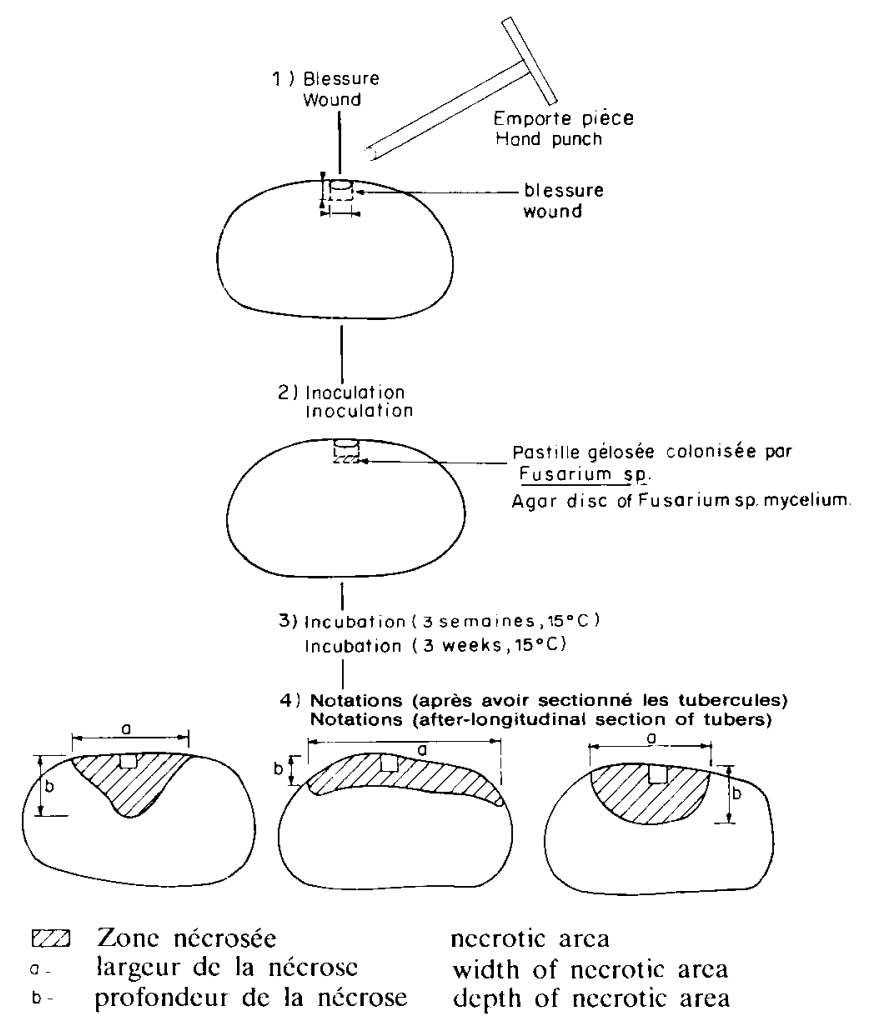

Figure 1

Méthode d'inoculation des tubercules de pomme de terre par les Fusarium et appréciation de la taille des nécroses.

Inoculation technique of potato tubers by Fusarium and evaluation of the rot-size.

\section{RÉSULTATS}

\section{Nature et fréquence des différents Fusarium isolés}

a) Nature des agents de Fusariose isolés

Trois espèces de Fusarium sont principalement représentées. Il s'agit de F. solani var. coeruleum, F. roseum (var. 

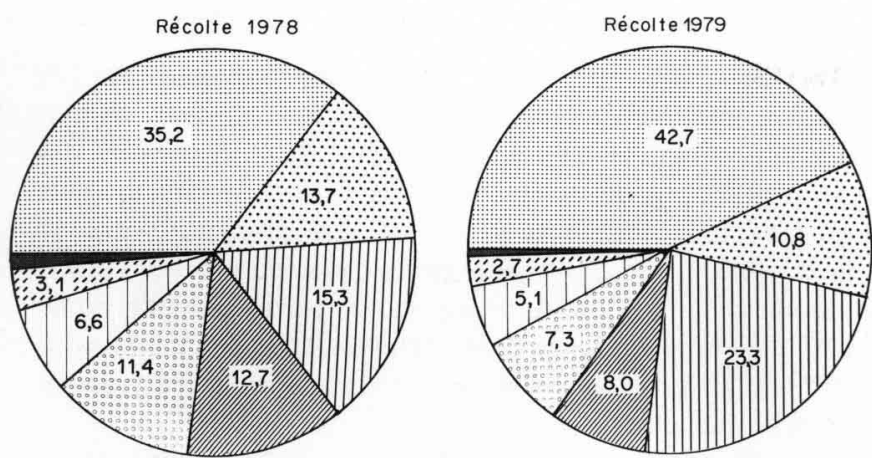

F.r. var. sambucinum

F.s. var. coeruleum

F.r. var. arthrosporioïdes

F.r. type $X$

F.r. var. graminearum

F.r. var. culmorum

F. oxysporum

Autres Fusarium

Figure 2

Fréquence des différentes espèces ou variétés de Fusarium isolées de pourriture des tubercules de pomme de terre récoltés en 1978 et 1979 (en p. 100).

Percentage of the different Fusarium species or varieties isolated from the rots of potato tubers lifted in 1978 and 1979.

sambucinum, var. arthrosporioides, var. graminearum, var. culmorum, type X) et $F$. oxysporum (Schlecht) Sn. et $\mathrm{H}$. Dans une très faible proportion des souches de $F$. roseum var. gibbosum (Wr.) Messiaen \& Cassini et de F. tricinctum ont également été isolées.

\section{b) Fréquence}

La fréquence relative des différents Fusarium varie peu entre les 2 années comparées (fig. 2). Ainsi, $F$. roseum var. sambucinum constitue l'agent pathogène le plus répandu puisqu'il représente entre 35 et 40 p. 100 de la flore fusarienne. Les autres Fusarium sont présents dans une plus faible proportion : $F$. solani var. coeruleum représente entre 11 et 13 p. 100 des espèces de Fusarium, F. roseum var. arthrosporioides 15 à 23 p. $100, F$. roseum var. graminearum et type $\mathrm{X}$ ont à peu près la même fréquence ( 8 à $12 \mathrm{p}$. 100). Enfin, $F$. roseum var. culmorum et $F$. oxysporum sont isolés dans peu de cas, respectivement 5 à 7 p. 100 et 3 p. $100 ; F$. roseum var. gibbosum et $F$. tricinctum représentent environ 0,5 p. 100 des espèces de Fusarium isolées.

\section{Agressivité des différentes espèces ou variétés de Fusarium isolées}

Le rôle des Fusarium comme agents saprophytes, susceptibles de s'introduire sur des lésions occasionnées par d'autres parasites, est bien connu, aussi avons-nous été amenés à réinoculer ces Fusarium à des tubercules de pomme de terre pour déterminer leur aptitude au parasitisme.

Dix isolats de chacune des espèces ou variétés de Fusarium, isolés précédemment sont inoculés sous forme de pastilles gélosées colonisées par du mycélium à 10 tubercules du cultivar «Bintje». Afin d'évaluer l'importance de la blessure dans le processus d'infection, l'inoculum est soit déposé sur le périderme des tubercules non blessés, soit introduit dans des cavités ménagées à la surface des tubercules. Les tubercules sont ensuite placés en atmosphère humide, à une température de $15^{\circ} \mathrm{C}$, pendant 3 semaines.

Après ce temps d'incubation, un feutrage mycélien s'est développé à la surface des tubercules non blessés mais

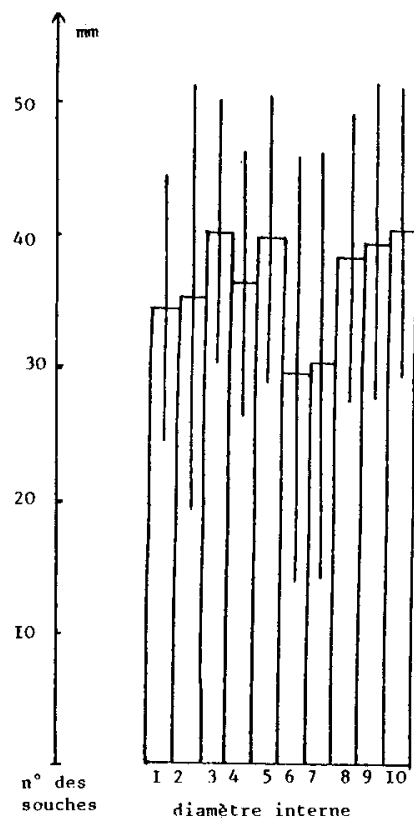

F. roseum var. oambucinum
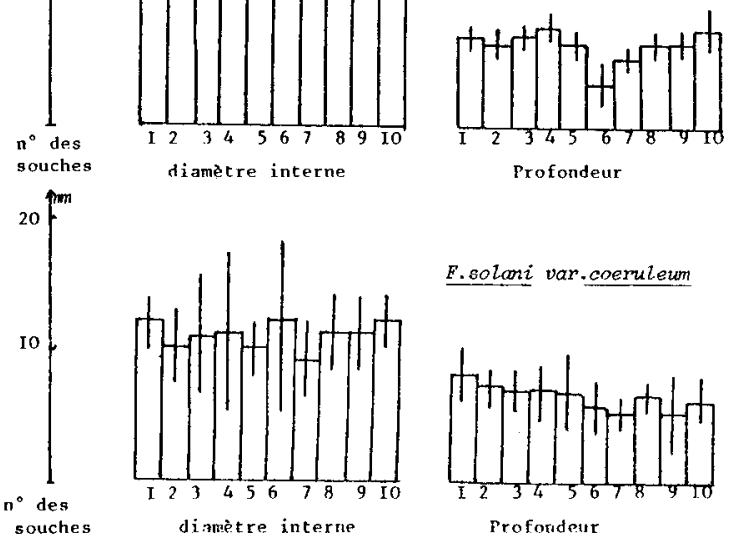

F.soloni var. coerulew
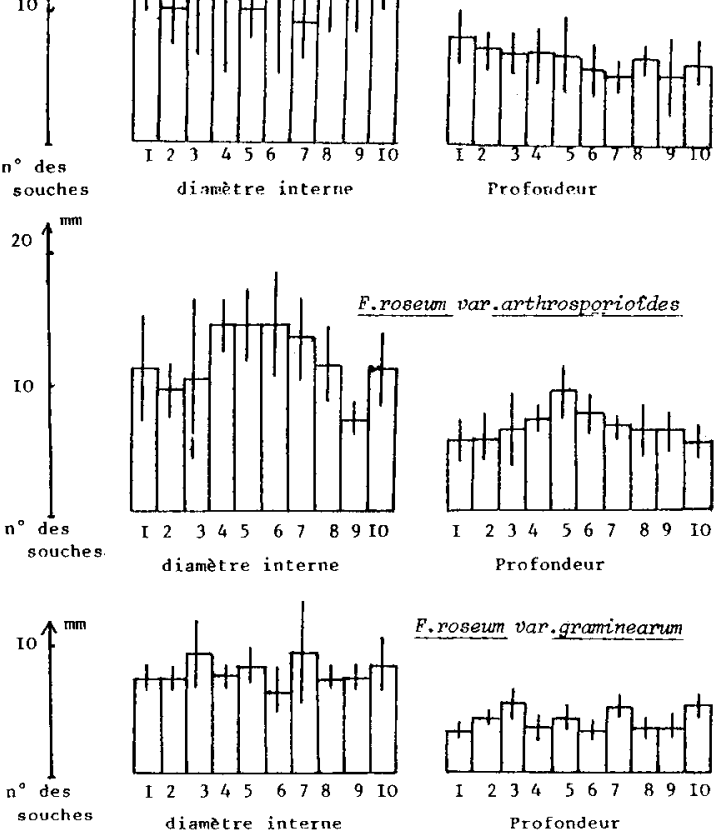

F.roseum var.graminearum
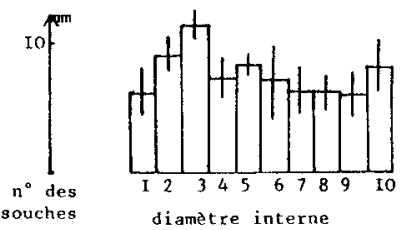

roseum type $x$

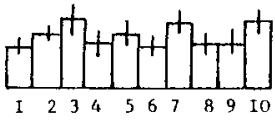

Profondeur
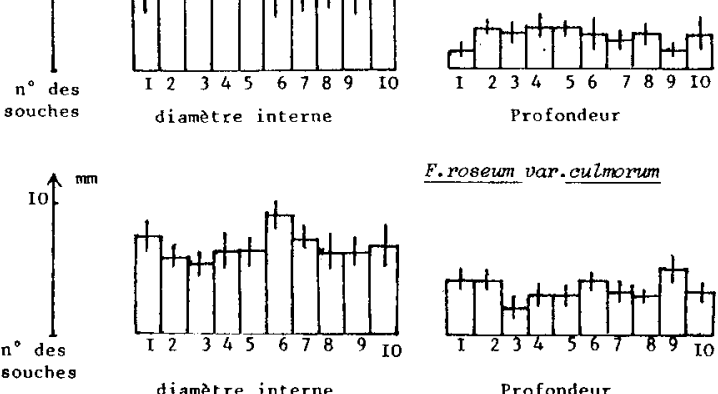

F.roseum var.culmorum

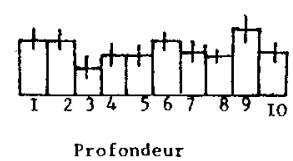

Figure 3

Agressivité de 10 souches de 6 espèces ou variétés de Fusarium sur tubercules de pomme de terre (exprimée par la taille de la largeur et de la profondeur de la nécrose en $\mathrm{mm}$ ).

Pathogenicity of 10 strains of Fusarium species or varieties on potato tubers (mean of width and depth of rot-mm). 
aucun signe de pourriture ne s'est manifesté, sauf chez les tubercules inoculés par $F$, roseum var. sambucinum. Les nécroses de petite taille sont alors localisées dans le tubercule à la base des germes développés pendant l'incubation. Il est vraisemblable que le champignon se soit introduit dans les tubercules grâce aux microblessures provoquées sur le périderme par l'émergence des germes.

Les tubercules blessés présentent, après incubation, des degrés d'attaque différents. On peut selon la gravité du symptôme (fig. 3), distinguer 3 groupes d'agents pathogènes (les 10 isolats de $F$. oxysporum et les 3 isolats de $F$. roseum var. gibbosum testés ne provoquant aucune nécrose sur les tubercules):

\section{a) Groupe très agressif}

Ce groupe se limite à la seule espèce, $F$. roseum var. sambucinum. Dès le $2^{\mathrm{e}}$ jour après l'inoculation, le parasite provoque des lésions dont l'extension entraîne une pourriture importante des tubercules. Il occasionne de loin les symptômes les plus graves puisque la largeur des lésions peut atteindre, en 3 semaines, 3 à $4 \mathrm{~cm}$. Le champignon ne semble pas pouvoir progresser à l'intérieur du tubercule puisque la nécrose reste relativement localisée au cortex. La variabilité entre les isolats est faible.

\section{b) Groupe moyennement agressif}

Ce groupe est constitué de $F$. solani var. coeruleum et de $F$. roseum var, arthrosporioides. Bien que les types de symptômes soient différents, la taille des nécroses occasionnées par ces 2 champignons varie entre 1 et $1,5 \mathrm{~cm}$ de largeur et atteint à peine $1 \mathrm{~cm}$ en profondeur. $F$. solani var. coeruleum, après une phase d'installation plus lente que $F$. roseum var. sambucinum, provoque un symptôme «en coin » typique, avec une limite diffuse entre les zones saine et nécrosée (BOYD, 1972) tandis que le contour de la pourriture occasionnée par $F$. roseum var. arthrosporioides est généralement plus net. Ce dernier parasite provoque, dans le tubercule, une large cavité dont les parois sont tapissées de mycélium. A ce groupe on doit également associer l'espèce $F$. tricinctum dont les 3 isolats testés provoquent des dégâts identiques à ceux de $F$. roseum var. arthrosporioides.

\section{c) Groupe peu agressif}

Ce groupe comprend $F$. roseum var. graminearum, $F$. roseum var. culmorum et $F$. roseum type X. Ces champignons provoquent dans les conditions de l'expérience, des pourritures peu développées, de 0,5 à $1 \mathrm{~cm}$ de largeur et de profondeur, extrêmement sèches. La pourriture qui se développe jusqu'au $4^{\mathrm{e}}$ jour après l'inoculation ne s'étend pas par la suite ; il se forme une cavité partiellement remplie d'une matière d'aspect farineux et séparée des tissus sains par une zone nécrosée, d'environ $1 \mathrm{~mm}$ de largeur, qui se détache facilement des tubercules.

Ainsi, il existe une grande différence dans l'aptitude de chacune de ces espèces ou variétés de Fusarium à provoquer des symptômes (fig. 4). Par contre, l'agressivité varie peu entre les isolats, au sein d'une même espèce ou variété de Fusarium. Il faut remarquer également que, parmi les mesures effectuées, la profondeur des lésions, qui est dans tous les cas peu développée, ne permet pas de distinguer les Fusarium étudiés. La mesure de la largeur des lésions permet une meilleure comparaison.

\section{Action de la température sur le développement des différentes espèces ou variétés de Fusarium}

Afin de préciser l'influence de la température sur le développement des pourritures, l'isolat le plus agressif, choisi à l'intérieur de chacune des espèces ou variétés de Fusarium précédemment citées, est inoculé à des tubercules du cultivar «Bintje». Vingt tubercules inoculés sont placés à $5,10,15,20,25$ et $30^{\circ} \mathrm{C}$ pendant 2 semaines. La mesure des largeurs des nécroses (fig. 5) permet de classer les Fusarium en 3 groupes en fonction de l'optimum thermique d'infection (la souche de $F$. oxysporum n'ayant pas provoqué de symptômes, quelles que soient les conditions de température).

\section{a) Groupe à optimum thermique élevé}

Ce groupe comprend $F$. roseum var, arthrosporioides, $F$. roseum var. graminearum et $F$. roseum var. culmorum. Ces Fusarium produisent des symptômes de pourriture sèche à $25-30^{\circ} \mathrm{C}$ mais seulement des nécroses incapables d'évoluer à

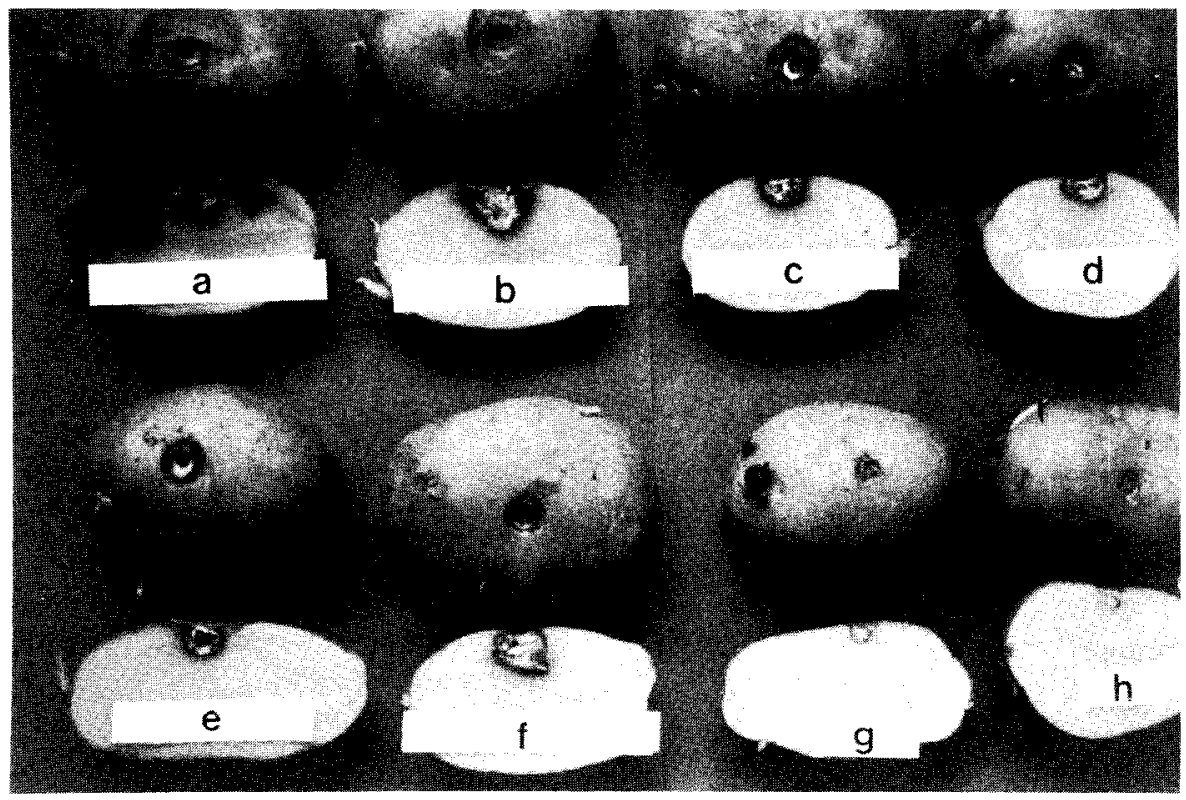

Figure 4

Tubercules du cultivar "Bintje» infectés par différentes espèces ou variétés de Fusarium après inoculation à $15^{\circ}$ pendant trois semaines.

Inoculation of potato tubers $(\mathrm{cV}$ "Bintje ") by different Fusarium species and varieties (incubation: 3 weeks, $15^{\circ}$ ).

a) F. roseum var sambucinum; b) $F$ solani var. coeruleum; c) $F$. roseum var. graminearum; d) $F$. roseum type $\mathrm{X}$; e) F. roseum var. culmorum ; f) F. roseum var. arthrosporioides $F$. oxysporum; g) Témoin. 


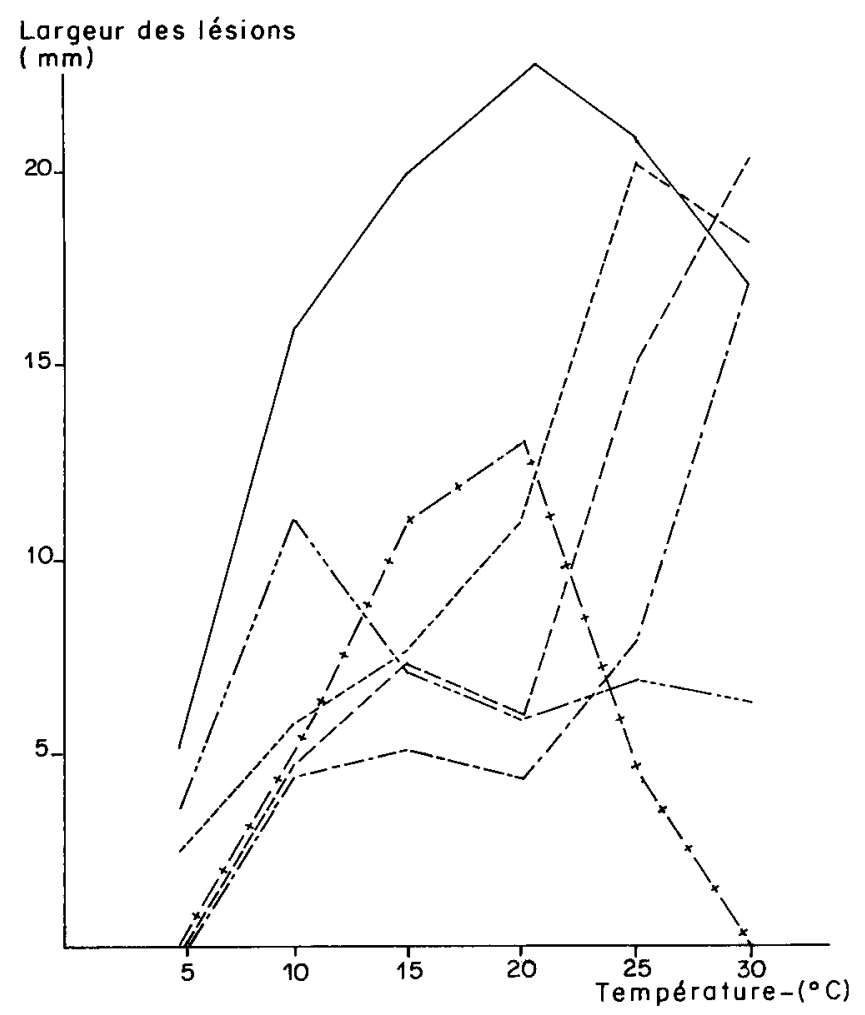

Figure 5

Influence de la température sur le développement des pourritures des tubercules de pomme de terre provoquées par 6 espèces ou variétés de Fusarium (exprimée par la largeur moyenne des lésions).

Action of the temperature on the dry rot of Fusarium species or varieties (width of the rot-mm).

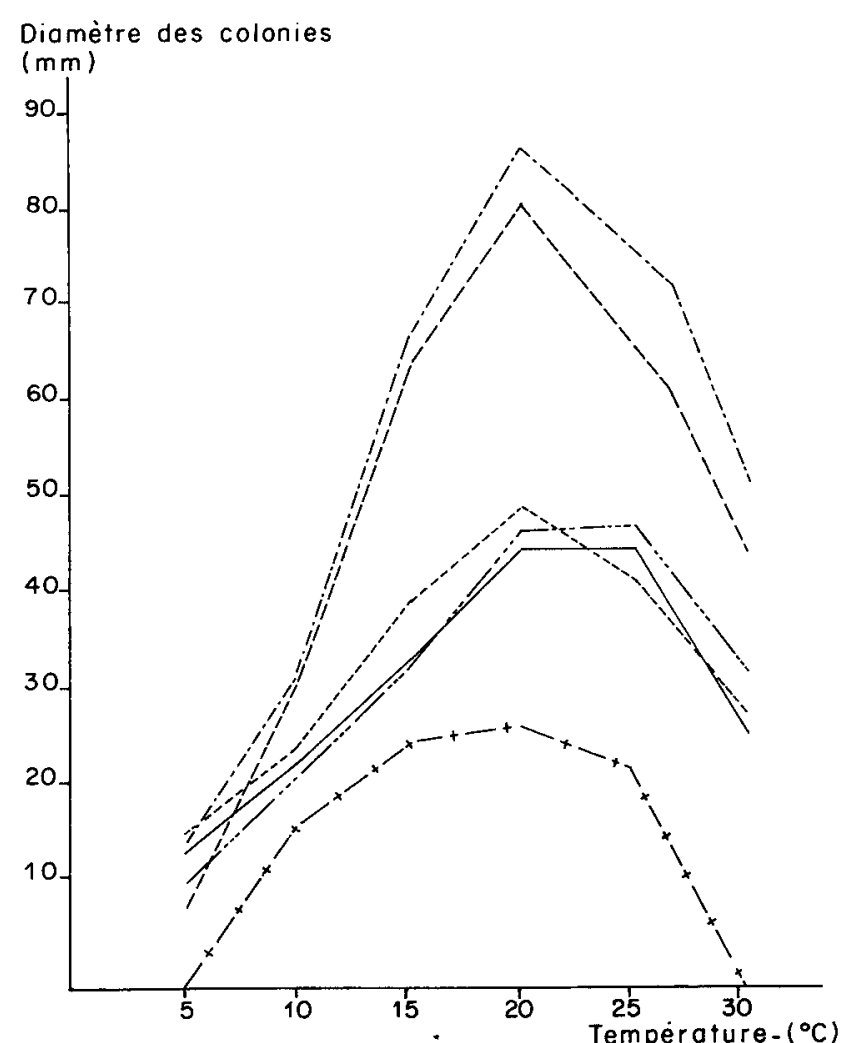

Figure 6

Influence de la température sur la croissance des colonies de 6 espèces ou variétés de Fusarium (exprimée par le diamètre moyen des colonies en $\mathrm{mm}$ après 6 jours de culture).

Action of the temperature on the growth of Fusarium species and varieties (mean diameter-mm- of cultures after 6 days on malt agar).

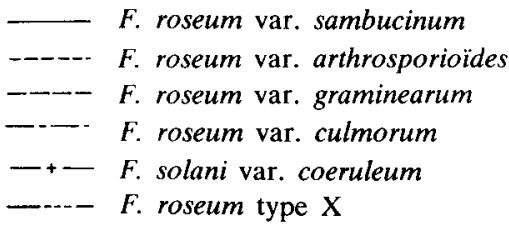

graves dégâts à $30^{\circ} \mathrm{C}$, température à laquelle leur croissance est sensiblement ralentie in vitro.

\section{CONCLUSION}

Ces résultats situent l'importance des différents Fusarium isolés des tubercules de pomme de terre en voie de pourriture.

Le nombre des espèces ou variétés de Fusarium susceptibles de provoquer actuellement des pourritures sèches laisse supposer que la flore pathogène fusarienne s'est sensiblement modifiée depuis les travaux de LANSADE (1950). Une évolution semblable est notée en Grande-Bretagne par MOORE (1945) qui remarque que $F$. avenaceum est apparu en 1943 bien après $F$. coeruleum. L'évolution des techniques culturales, des types d'assolement ainsi que l'apparition de nouveaux cultivars de pomme de terre ne sont peut être pas étrangers à cette évolution.

Les tubercules peuvent être soumis, avant ou après la phase de conservation au froid $\left(4{ }^{\circ} \mathrm{C}\right)$, à des températures 
qui favorisent le développement des pourritures à Fusarium. Ces températures comprises entre 15 et $25^{\circ} \mathrm{C}$ se rencontrent, soit dès la récolte, lorsque les tubercules sont stockés pendant 2 à 3 semaines à température ambiante afin d'activer le phénomène de cicatrisation des blessures occasionnées lors de la récolte, soit avant la plantation suivante, lors du transport ; cette phase est surtout importante pour le plant destiné à l'exportation.

Deux Fusarium occasionnent, sous nos climats et de façon constante, la pourriture des tubercules: F. roseum var. sambucinum, qui est à la fois le plus fréquent et le plus agressif des Fusarium isolés, et $F$. solani var. coeruleum.

Un $3^{c}$ Fusarium: F. roseum var, arthrosporioides, que l'on isole aussi fréquemment que $F$. solani var. coeruleum entraîne, lorsque la température dépasse $20^{\circ} \mathrm{C}$, des pourritures importantes (fig. 7).

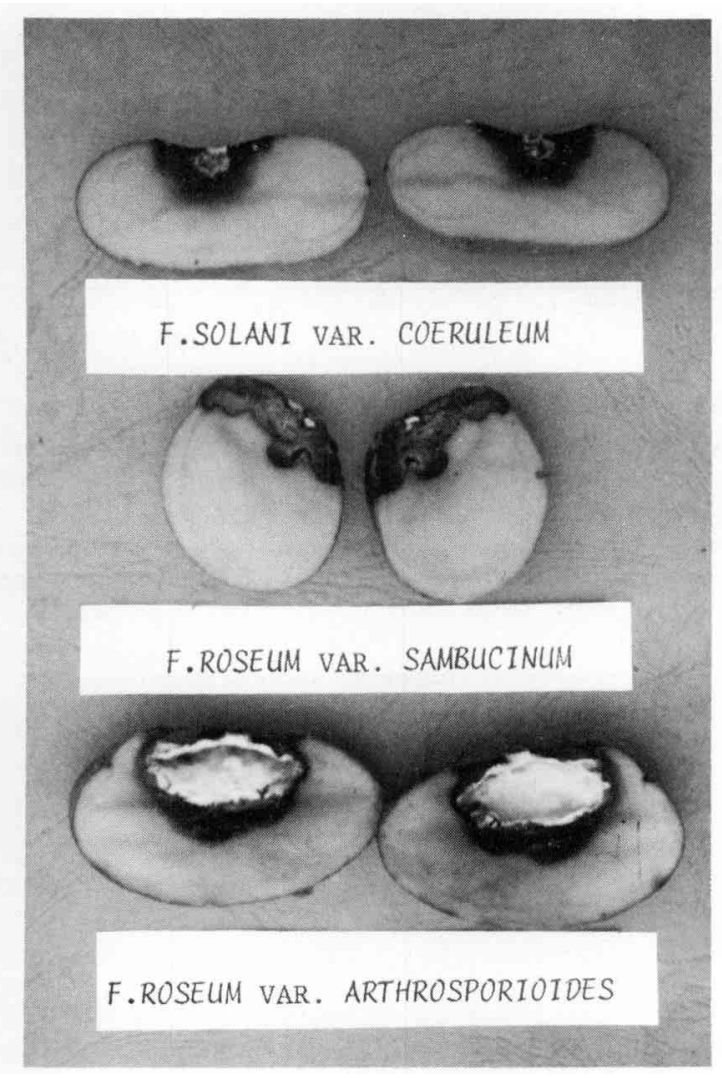

Figure 7

Tubercules du cultivar "Bintie " infectés par les trois espèces ou variétés de Fusarium les plus importantes en France. (Les tubercules inoculés par F. solani var. coeruleum et par F. roseum var. sambucinum ont été incubés à $15^{\circ} \mathrm{C}$ pendant 3 semaines; les tubercules inoculés par $\mathrm{F}$. roseum var. arthrosporioides ont été incubés à $25^{\circ} \mathrm{C}$ pendant trois semaines).

Inoculation of potato tubers ( $\mathrm{cv}$ *Bintje $»)$ by 3 predominating species of varieties in France. (Incubation: 3 weeks, $15^{\circ} \mathrm{C}$ for $\mathrm{F}$. roseum var. sambucinum and $\mathrm{F}$. solani var. coeruleum and 3 weeks, $25^{\circ} \mathrm{C}$ for $\mathrm{F}$. roseum var. arthrosporioides).
Ces 3 Fusarium, par leurs exigences thermiques assez basses, semblent particulièrement adaptés aux conditions climatiques de notre pays ; ce sont d'ailleurs eux qui ont la fréquence d'isolement la plus forte.

Si $F$. roseum var. graminearum et $F$. roseum var. culmorum, faiblement représentés dans la flore fusarienne, sont susceptibles d'occasionner des pourritures sérieuses à partir de $25^{\circ} \mathrm{C}, F$. roseum type $\mathrm{X}$ n'entraîne qu'une faible nécrose des tubercules. L'incapacité de $F$. oxysporum à s'installer sur les tubercules laisse supposer que son rôle dans les attaques est nul. Les souches de ce champignon isolées en France à partir de tubercules sont sans doute différentes de celles étudiées en Pologne par RATUSZNIAK \& PIOTROWSKA (1978) qui, elles, provoquent une pourriture.

La phase d'installation des différents Fusarium dans les tubercules peut se réaliser à température assez basse ( 5 ou $10^{\circ} \mathrm{C}$ ) mais le devenir des nécroses n'est pas le même selon les espèces ou variétés de Fusarium. Ainsi, pour $F$. roseum var. culmorum, $F$. roseum var. graminearum, $F$. roseum type $\mathrm{X}$ et, à un moindre degré, pour $F$. roseum var. arthrosporioides, la possibilité de provoquer des dégâts importants dépend étroitement de la température : l'existence de nécroses qui n'évoluent pas à des températures inférieures à $15^{\circ} \mathrm{C}$ laisse supposer une modification des relations hôte-parasite selon les températures. Si les conditions de température sont favorables, ces champignons provoquent une pourriture sèche typique (sauf pour $F$. roseum type $\mathrm{X}$ dont le rôle parasitaire n'est pas clairement établi). Il n'en est pas de même chez $F$. solani var. coeruleum et $F$. roseum var. sambucinum, car même à 5 ou $10{ }^{\circ} \mathrm{C}$, ces champignons progressent dans les tubercules, mais très lentement, entraînant inexorablement leur pourriture (BOYD, 1972 ; LANSADE, 1950).

Ces résultats montrent que la flore pathogène responsable de la pourriture sèche des tubercules est très complexe et que, sous l'action des conditions de milieu, telle ou telle espèce de Fusarium est plus particulièrement favorisée. Obtenus après inoculation du seul cultivar «Bintje », ils ne sont toutefois pas généralisables à l'ensemble des cultivars de pomme de terre chez lesquels des auteurs ont déjà mentionné l'existence de réactions plus ou moins spécifiques avec certains Fusurium; ainsi récemment, CoRSINI et PAVEK (1979) ont montré que la résistance d'un hybride ou d'un cultivar à $F$. roseum var. sambucinum était totalement indépendante de la résistance à $F$. solani var. coeruleum.

Le facteur variétal ainsi que d'autres facteurs liés à la plante (âge physiologique, rapidité de cicatrisation) ou au parasite (forme et importance de l'inoculum) seront abordés lors d'un prochain article.

Reçu le 24 mai 1980. Accepté le 17 juin 1981.

\section{REMERCIEMENTS}

Les auteurs tiennent à remercier Monsieur MEssiaEn pour l'aide précieuse qu'il leur a apportée dans la détermination des Fusarium.
Boissonnet-Menes Marcelle, Lecoq H., 1976. Transmission de virus par fusion de protoplastes chez Pyricularia oryzae. Physiol. vég. 14, 2, 251-257.

Booth C., 1971. The genus Fusarium. Kew England: Commonwealth Mycol. Inst. 237 p.
Boyd A. E. W., 1972. Potato Storage diseases. Rev. Plant Pathol, 51, 5, 297-321.

Corsini D., Pavek J., 1979. Inheritance of potato tuber Fusarium dry rot resistance. Phytopathology, 69, 8, 914-915. 
Lansade M., 1950. Recherches sur la Fusariose ou pourriture sèche de la pomme de terre, Fusarium coeruleum (Lib.) Sacc. Ann. Epiphyt. 1, 157-207.

MacKee R. K., 1954. Dry-rot disease of the potato. VIII. A study of the pathogenicity of Fusarium coeruleum (Lib.) Sacc. and Fusarium avenaceum (Fr.) Sacc. Ann. appl. Biol. 41, 3, 417-434. Messiaen C. M., Belliard-Alonzo L., Barrière Y., de la Tullaye B., 1976. Etude qualitative des Fusarium roseum dans les sols des environs de Versailles, sous diverses rotations ou associations végétales. Ann. Phytopathol., 8, 3, 269-281.

Messiaen C. M., Cassini R., 1968. Recherches sur les Fusarioses. IX. La systématique des Fusarium. Ann. Epiphyt., 19, 3, 387-454.

Moore F., Joan, 1945. A comparison of Fusarium avenaceum and Fusarium coeruleum as causes of wastage in stored potato tubers. Ann. appl. Biol., 32, 304-309.

Ou, 1972. Rice diseases. C.M.I. Kew, Surrey, England, 368 p.
Pett B., Gotz J., 1978. Distribution of potato-pathogenic Fusaria with particular reference to the territory of the G.D.R. Symp. Soz. Länder Inst. Kartoffelforsch. Gross. Lüsewitz Akad. Landwirtschafiswissench. D.D.R. Rostock, 1-7 nov. 1976. Akad. landwirtscheftswiss. Berlin, Tagungsber, $\mathrm{n}^{\circ} 157,45-53$.

Ratuszniak, Sas Piotrowska, 1978. Tests for the determination of the relation between a few species of fungi of thegenus Fusarium in mixed infection. Symp. Soz. Länder. Inst. Kartoffelforsch. Gross. Lusewitz. Akad. Landwirtschaftswissench. D.D.R. Rostok, 17 nov. 1976. Akad. Landwirtschaftswiss. Berlin, Tagungsber, $\mathrm{n}^{\circ} 157,45-53$.

Tivoli B., Jouan B., L'Hostis A., Sanson Marie-Thérèse, Lemarchand E., 1979. Influence de l'aménagement des locaux de conservation des tubercules de pomme de terre sur leur niveau de contamination et sur l'efficacité de la désinfection. Sci. agron., Rennes, 1979, 20-25. 